\title{
COMBINATORIAL HYPERMAP REWRITING
}

\author{
Eric SOPENA \\ Département Informatique \\ I.U.T. "A", Université de Bordeaux I \\ F-33405 Talence
}

\begin{abstract}
Combinatorial hypermaps may be viewed as topological representations of hypergraphs. In this paper, we introduce a hypermap rewriting model, based on a purely combinatorial formulation of the rewriting mechanism. We 11 lustrate this definition by providing a hypermap gramar which generates the set of all connected planar maps We also investictate a special kind of hypermap grammars, the H-grammars, for which we eive a Pumping Theorem enlightening the combinatorial structure of the Eenerated hypermap languages
\end{abstract}

\section{o. INTRODUCTION}

The appearance of graph grammars, in the early 70's, was essentially motivated by practical concerns in picture processing (recognition of chromosomes or manuscript letters). They were influenced by many application areas such as databases, compiling techniques, abstract data types, distributed systems or the development of biological cells [2,6]. The diversity of applicability domains of such grammas bives rise to the definition of many graph rewriting systems, which deal with rather different kinds of problems. Theoretical research essentially attempts to retrieve the main results from formal language theory. Some models now possess a well-developed mathematical theory, such as the algebraic approach of thrig [5] or the NLC (Node-Label-Controlled) grammars of Janssens and Rozenberg [8].

Most modeis of Graph grammars are based on a graph, or subsraph, rewriting operation and the iteration of such a process. Due to the very structure of graphs, the rewriting mechanism, and especially the "embedding" operation, is presented in a more complex, and sometimes less rigorous way, than in formal language theory. The recent results of Bauderon and Courcelle [1] provide a simplification of the graph rewriting mechanism, based on a representation of sraphs by algebraio expressions.

One must point out that, in adition to problems issued from formal language theory colassification of graph languages, properties of derivation sequences, decidability problems, the graph grammar theoretician deals with many problems related to the structure of generated Eraphs (planar graphs, Hamiltonian graphs, conneoted graphs).

Representation of graphs on surfaces takes a prominent part in graph theory. Meanhile, we notice the lack of rewriting systems dealing with topological maps, even anterior to the concept of graph iseefor 
example the four colour problem). In this paper, we attempt to fill this gap by defining a map (or more senerally a hypermap) generating system based on a combinatorial representation of them, introduced by Edmonds [4]. Combinatorial hypermaps may be viewed as topological representations of hypergraphs, and are defined by a pair $(\sigma, \alpha)$ of permutations acting on a finite set of darts [3] (darts represent pairs of incident edges and vertices; permutation $\sigma$ (resp. $\alpha$ ) gives an order to the edges (resp. vertices) incident to a given vertex (resp. edge)). The interest in such a model essentially lies in the simplicity of the rewriting mechanism, expressed in a strictly combinatorial way, as a product of permutations.

In the first seotion, we introduce the main objects of our proposal: hypermaps and hypergraphs, and point out the links between them by notions of representation of hypereraphs and the underlying hypergraph of a given hypermap.

The second section is devoted to the definition of our hypernap rewriting model. A production $p$ is made of a pair (L,R) of labelled hypermaps. A labelled hypermap $H$ can then be rewritten by application of production $p$ in three steps: localise an occurrence $\mu L$ of left-hand-side $L$ in $H$, isolate each dart of occurrence $\mu L$ and then glue them together using the "scheme" given by right-hand-side $R$ (this step may introduce new darts). The resulting hypermap is expressed as a combinatorial product of the form: $\xi D \otimes \mu L^{-1} \otimes H$.

In the third section we illustrate these definitions by providing a hypermap grammar which generates the set of all connected planar maps. The last section deals with a special kind of hypermap grammars: the H-grammars. Bach production realize the rewriting of a full hyperedge. We show that these grammars may be considered as context-Free hypermap grammars and provide a theorem on the combinatorial structure of generated languages, generalizing a well-known result (Pumping Lemma) from formal language theory.

Due to the limitation of space, we will omitothe proofs which can be found in [9].

\section{BASIC DEFINITIONS}

In this work, we will denote by $[n]$ the set $\{1,2, \ldots, n\}$, and by $\left[n_{1}, n_{g}\right]$ the set $\left\{n_{1}, n_{1}+1, \ldots, n_{2}\right\}$.

Let $B$ be a finite set; a permutation $\beta$ acting on $B$ is a bijection from $B$ to itself: A cycte of $\beta$ is a sequence $\left(b_{1}, \ldots . b_{k}\right.$ ) of elements of $B$ such that $B b_{k}=b_{1}$ and $\forall i \in[k-1], B b_{i}=b_{1+1} ;$ the set $\left\{b_{1}, \ldots, b_{k}\right\}$ is called an orbit of $B$.

Let $A$ be a subset of $B$; the restriction of $B$ to $A$, denoted by $B$, is the permutation acting on $A$ defined by: $\forall a \in A, B_{1 A} \alpha=\beta^{k} \alpha$ where $k$ is the smallest positive integer such that $\beta^{k} a \in A$.

Let $C$ be a set containing $B$; the extension of $B$ to $C$, denoted by ext $(B)$ is the permutation acting on $C$ defined by: $\forall c \in C$, if $c \in B$ then 
$e x t_{c}(\beta) C=B C$ else extc $(\beta) C=0$. Whenever the set $C$ is given by context, we simply write $\beta$ to denote exto $(\beta)$.

Example: Let $\beta$ be the permutation defined on $B=[6]$ by:

$$
B=(1,3)(2,4,6)(5) \text {, }
$$

thus we have:

$$
\beta_{1,4\}}=(1,3)(2,4) \text { and } \operatorname{ext}_{18,}(\beta)=(1,3)(2,4,6)(5)(7)(8)
$$

A hypermap is a triple $H=(D, \sigma, \alpha)$ where $D$ is a finite set of arts, $\sigma$ and $\alpha$ are two permutations over $D$. The orbits of $\sigma$ (resp. $\alpha$ ) are called the vertices (resp. hyperedges) of the hypermap. Let $\alpha \sigma$ be the permutation defined by $\alpha \sigma d=\alpha(\sigma(d))$ for each $d$ in $D$. The orbits of $\alpha \sigma$ are called the faces of the hypermap.

The cardinality \#D of set $D$ is the order of the hypermap. We call the degree of a vertex (resp. hyperedge, face) the number of darts belonging to this vertex (resp. hyperedge, face). A vertex and a hyperedge are said to be incident if they have one dart at least in common.

If the group $G_{\sigma \alpha}$ generated by $\langle\sigma, \alpha\rangle$ is transitive, we say the hypermap $H$ is connected (i.e, $\forall a_{1}, a_{z} \in D, \exists \tau \in G_{\alpha \alpha} / a_{1}=\tau d_{z}$ )

A hypermap $H=(D, \sigma, \alpha)$ is called a map iff the permutation $\alpha$ is a fixpoint free involution (i,e. $\forall a \in D, \alpha d \neq a$ and $\alpha^{2}(d)=d$ ).

Let $H=(D, \sigma, \alpha)$ be a hypermap. $Q_{0}$ and $d_{p}$ two darts of $D ;$ a chain from $d_{0}$ to $d_{p}$ is a sequence $d_{0}, d_{1}, \ldots, d_{p}$ of darts such that: $\forall i \in[p], d_{i}=$ $\sigma d_{i-1}$ or $a_{i}=\alpha d_{i-1}$. Thus, a hypermap is connected iff for every pair $\left(d, d^{\prime}\right)$ of darts there exists $a$ chain from $d$ to $d^{\prime}$. Moreover, the relation $\mathscr{R}$ defined by: $d \mathscr{R} d^{\prime}$ iff there exists a chain from $d$ to $d^{\prime}$ is an equivalence relation. Let $C$ be an equivalence class of $R$; the hypermap $\left(C, \sigma_{1}, \alpha_{10}\right)$ is a connected component of $H$. We will denote by $C C$ (H) the set of all connected components of $H$.

The genus of a connected hypermap $H$ is the quantity:

$$
g(H)=1+1 / z[\# D-z(\sigma)-z(\alpha)-z(\alpha \sigma)],
$$

where for any permutation $\beta, z(\beta)$ denotes the number of cycles of $\beta$.

The genus of a general hypermap is then: $g(H)=\max \{g(C) / C \in C C(H)\}$. A hypermap with genus zero is said to be planar. Thus, a hypermap is planar iff each of its connected components is planar.

In order to ensure homogeneity, every hypermap of order $n$ will be defined over $D=[n]$.

A hypergraph is a pair $K=(V, E)$ where $V$ is a finite set of vertices and $E$ a finite set of edges. An edge is a family $e=\left\{v_{1}, \ldots, v_{p}\right\}$ of elements of $V$ (note that the same vertex may appear twice or more).

Let $K=(V, E)$ be a hypercraph; without loss of generality, one can suppose that each vertex $v$ of $V$ belongs to one edge $e$ of $E$ at least. From $K$, we define a set of darts as follows: for any vertex $v$ and any edge e incident to $v$, let $k$ be the number of occurrences of $v$ in $e$; then we define $k$ darts denoted by $\langle v, e\rangle_{1}, \ldots,\langle v, e\rangle_{k}$. Thus, $V$ and $E$ determine two partitions over $D$ whose classes are respectively associated with the vertices and edges of $K$. By giving an arbitrary 
order to each of these classes, we define two permutations over $D$. Let $\sigma$ and $\alpha$ be these two permutations; we will say that the hypermap $H=(D, \sigma, \alpha)$ is a representation of hypergraph $K$.

Example: Figure 1 shows a hypergraph $K(1, a)$ and a hypermap $H(1, b)$ which is a representation of $K$ (vertices of $H$ are drawn as circles with darts on their circumferences; for vertices and hyperedges, positive orientation has been choosen as clockwise).

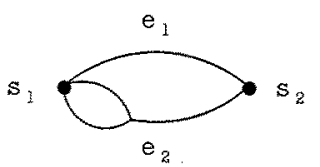

$e_{1}=\left\{s_{1}, s_{2}\right\}$ $e_{2}=\left\{s_{1}, s_{1}, s_{2}\right\}$

(a)

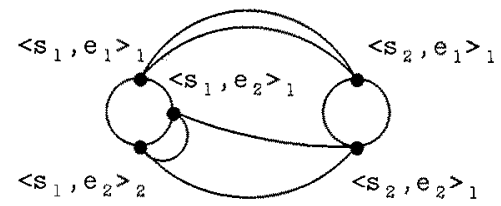

(b)

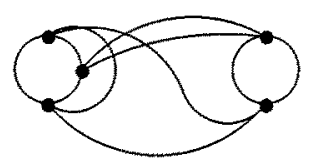

(c)

- figure 1-

Conversely, let $H=(D, \sigma, \alpha)$ be a hypermap; the underlying hypergraph $K$, denoted by Und(H), is built in the following way: orbits of $\sigma$ are the vertices of $K$; the edges are obtained from the orbits of $\alpha$ by replacing every dart by the vertex it belongs to.

If $H$ is a connected hypermap, the underlying hypergraph Und( $H)$ is clearly connected. The underlying hypergraph of a map is a graph. The underlying hypergraph of a planar hypermap is planar, but a hypermap which is a representation of a planar hypergraph is not necessarily planar (figure 1.0 shows a non planar representation of hypergraph $K$ from figure l.a).

Let $\Sigma$ be a non-empty finite set of labels. A labelzed hypermap over $\Sigma$ is a 4-tuple $H=(D, \sigma, \alpha, \lambda)$ such that: $H=(D, \sigma, \alpha)$ is a hypermap and $\lambda$ a mapping from $D$ to $\Sigma$ called the labeliting function. The set of all labelled hypermaps over $\Sigma$ will be denoted by $\mathrm{H}_{\Sigma}$. A hypermap language is then a subset of $\mathrm{H}_{\Sigma}$.

Let $H_{1}=\left(D_{1}, \sigma_{1}, \alpha_{1}, \lambda_{1}\right)$ and $H_{2}=\left(D_{2}, \sigma_{2}, \alpha_{2}, \lambda_{2}\right)$ be two hypermaps of $H_{2}$. We say that $H_{1}$ and $H_{2}$ are isomorphic, denoted by $H_{1} \sim H_{2}$, if there exists a bijection $\mu$ from $D_{1}$ to $D_{2}$ such that: $\alpha_{2}=\mu \alpha_{1} \mu^{-1}, \sigma_{2}=\mu \sigma_{1} \mu^{-1}, \lambda_{2}=\lambda_{1} \mu^{-1}$. For such hypermaps, we will write $H_{2}=\mu H_{1}$.

Let $H=(D, \sigma, \alpha, \lambda)$ be an element of $H_{\Sigma}$, and $A$ a subset of $D$; the restriction of $H$ to $A$, denoted by $H_{1 A}$, is the hypermap $H^{\prime}=\left(D^{\prime}, \sigma^{\prime}, \alpha^{\prime}, \lambda^{\prime}\right)$ Eiven by: $D^{\prime}=A, \sigma^{\prime}=\sigma_{1 A}, \alpha^{x}=\alpha_{1 A}$ and $\lambda^{\prime}=\lambda_{I_{A}}$.

Let $H_{1}$ and $H_{z}$ be two hypermaps of $H_{z}$. We will say that $H_{z}$ is a subhypermap of $H_{1}$ if $f$ there exists a subset $A_{1}$ of $D_{1}$ such that $H_{2}=H_{11 A_{1}}$. 
The combinatorial product of two hypermaps, defined below, will enable us to give a very simple expression of hypermap rewriting mechanism.

Let $H_{1}=\left(D_{1}, \sigma_{1}, \alpha_{1}, \lambda_{1}\right)$ and $H_{2}=\left(D_{2}, \sigma_{2}, \alpha_{2}, \lambda_{2}\right)$ be two elements of $H_{\Sigma}\left(D_{1}\right.$ and $D_{z}$ rot necessarily disjoint). We define the hypermap $H^{3}=H_{2} \otimes H_{2} \quad a s$ follows: $\quad D^{\prime}=D_{2} \cup D_{g}, \quad \sigma^{3}=\sigma_{2} 0 \sigma_{2}, \quad \alpha^{\prime}=\alpha_{1} o \alpha_{2}$, if $d \in D_{1}$ then $\lambda^{\prime} d=\lambda_{1} a$ else $h^{2} d=\lambda_{g} d$.

Let $H$ be a hypermap; we will denote by $H^{-1}$ the hypermap defined by $H^{-1}=\left(D, \sigma^{-1}, \alpha^{-1}, \lambda\right)$. Note that $H^{-1}$ is the unique hypermap verifying HQH $=H^{-1} \otimes H=I$, with $I=(D, I d, I d, \lambda)$ where Id denotes the identity over $D$ (hypermap $H^{-1}$ may be viewed like hypermap H but with counterclockwise orientation).

\section{HYPERMAP GRAMMARS}

In this section, we introduce the concept of hypermap Eramars, based on a strictly combinatorial formulation of the rewriting mechanism.

2.1 Definition: A hypermap grammar is a 4 -tuple $G=\langle\Sigma, \Delta, P, Z\rangle$ where $\Sigma$ is a finite non-empty set (the total atphabet), $\Delta$ a non-empty subset or $\Sigma$ (the terminal aiphabet), $P \subset H_{\Sigma} \times H_{\Sigma}$ the set of productions and $Z \in H_{\Sigma}$ the $a x i o m$.

Let $p=(\tilde{L}, R)$ be aroduction; the hypermap $L$ (resp. R) is called lefthand-side (resp. pight-hand-side) of production $p$ and is denoted by LHS (p) (resp. RHS (p)). In order to simplify the derivation concept, we will assume that $D_{\mathrm{L}} C D_{\mathrm{R}}$. (i.e. in each derivation step, all darts are preserved).

2.2 Definition: Let $G=\langle\Sigma, \Delta, P, Z\rangle$ be a hypermap grammar ; a derivation step is a 5 -tuple $\left\langle H, p, \mu, \xi, H^{\prime}\right\rangle$ verifying:

(2.2.1) $H=(D, \sigma, \alpha, \lambda)$ and $H^{\prime}=\left(D^{\prime}, \sigma^{\prime}, \alpha^{\prime}, \lambda^{\prime}\right)$ are elements of $H_{z}$;

(2.2.2) $p=(L, R)$ is a production of $P$,

(2.2.3) $\mu$ is an injective mapping from $D_{k}$ to $D$ suoh that $\mu L$ is a subhypermap of $H$,

(2.2.4) $\xi$ is a bijection from $D_{\mathrm{R}}$ to $D^{*} \backslash D \cup \mu D_{\mathrm{L}}$ such that $\xi_{1 \mathrm{DL}}=\mu$,

(2.2.5) $H^{\prime}=\xi D \otimes H L^{-1} \otimes H$.

We will say that $H$ dipeotby derives $H^{\prime}$ and denote it by $H_{\mathrm{p},} H^{\prime}$ Whenever the values of $p$, Hare of no importance we simply note H $\rightarrow H H^{\prime}$.

Interpretation: The rewriting of $z_{\text {th }}$ onto $H^{2}$ by applying production $p=(L, R)$ may be decomposed in the following way:

Step 1: find an occurrence $\mu$ L of $L H S(p)$ in $H$ (condition 2.2.3),

Step 2: define a bijection $\xi$ which associates dart 1 (resp. 2,3,..) of $R$ with dart $\mu(1)$ (resp. $\mu(2), \mu(B), \ldots$ ) of occurrence $\mu L$ (condition 2.2.4),

Step 3: $H^{\prime}$ is then obtained by the combinatorial product $\xi D \otimes \mu L^{-1} \otimes H$. 
This product can be interpreted as:

i) $\mu L^{-1} \otimes H$ : "isolate" in $H$ each dart of $\mu L$,

ii) $\xi D \otimes\left(\mu L^{-1} \otimes H\right):$ "glue" different portions together using the scheme given by RHS $(p)$.

Note that according to the choice of $\xi$, we obtain different hypermaps which are isomorphic.

2.3 Example: Figure 2 shows a production $p$ and two hypermaps $H$ and $H^{\prime}$ $\overline{\text { such that } H} \mapsto H^{\prime}$ by applying production $p$ (hypermap $\mu L^{-1} \otimes H$ is given as an intermediate step of the derivation).
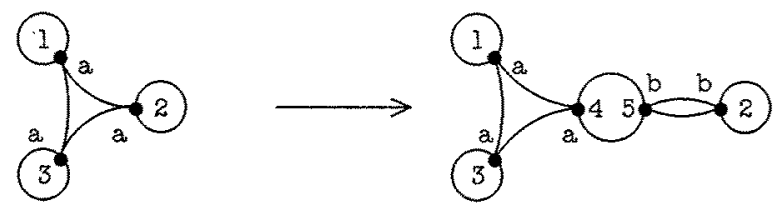

production $p=(I, R)$

$\mu L^{-1} \otimes \mathrm{H}$

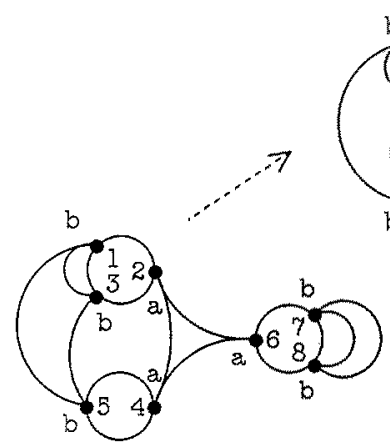

$\mathrm{H}$

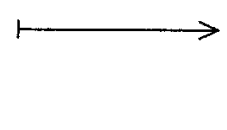

- figure 2-

2.4 Definition: Let $G=\langle\Sigma, \Delta, P, Z\rangle$ be a grammar, $H=(D, \sigma, \alpha, \lambda)$ and $H^{\prime}=\left(D^{\prime}, \sigma^{\prime}, \alpha^{\prime}, \lambda^{\prime}\right)$ two elements of $H_{\Sigma}$; we will say that $H$ derives $H^{\prime}$, denoted by $H^{*} \rightarrow H^{\prime}$, if either $H=H^{\prime}$ or there exists a sequence $H_{0}, H_{1}, \ldots, H_{t}$ of hypermaps such that: $H_{0}=H, H_{t}=H^{\prime}$, and $\forall i \in[t], H_{i-1} \mapsto H_{i}$. The language generated by $G$ is then the set:

$$
L(G)=\left\{H \in H_{\Delta} / \exists H_{I} \in H_{\Delta}, Z \mapsto * \rightarrow H_{1} \text { and } H \sim H_{I}\right\} \text {. }
$$

We call $H_{\Delta}$-tanguage all subset $L$ of HA such that there exists a hypermap grammar $G$ verifying $L(G)=L$. We will denote by LH. the set of al1 $H_{\Delta}-1$ anguages. 


\section{EXAMPLES}

In this section, we illustrate the previous concepts by providing two hypermap erammars which generate respectively the set of all planar maps with only one vertex, and the set of all connected planar maps.

3.1 Fxample (planar maps with only one vertex):

Let us consider the grammar $G=\langle\Sigma, \Delta, P, Z\rangle$ defined by:

1. $\Sigma=\Delta=\{a\}$,

2. $P=\{p\}$ with $p=(L, R)$ given by:
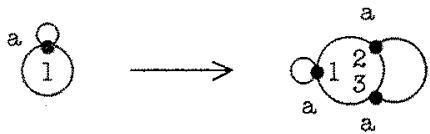

3. $a x i o m z$ is

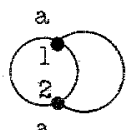

a.

Let $M$ be a planar map with only one vertex; applying production consists in choosing a dart $d$ in $M$ and adding a loop made af the two darts created by $p$ (this loop "follows", in the sense of permutation 0 , dart $a$ in the resulting hypermap $M^{\prime}$ ). Applying $p$ clearly preserves planarity and the number of vertices. Moreover we have:

3.2 Proposition: L(G) is exactly the set of all planar maps with only one vertex

Figure 3 shows an example of derivation sequence (darts marked e denote darts of occurrence $\mu L$, dotted edges denote created edges).

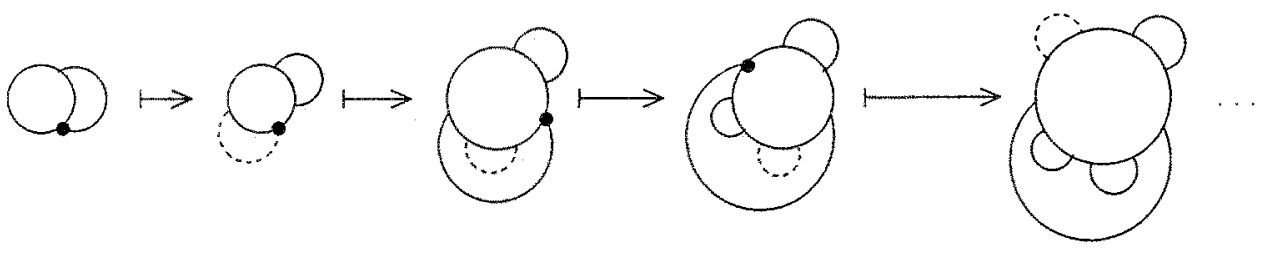

- figure 3 -

3.3 Example (connected planar maps):

Let us consider the grammar $G=\langle\Sigma, \Delta, P, Z\rangle$ defined by:

1. $\Sigma=\{a, b\}, \Delta=\{a\}$,

2. $P=\left\{p_{1}, p_{2}, p_{3}, p_{4}, p_{3}\right\}$ where $p_{1}, p_{2}, p_{3}, p_{4}, p_{3}$ are given by:

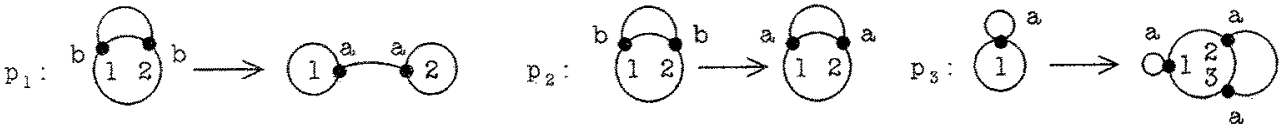



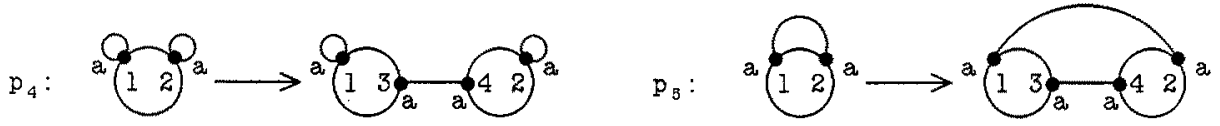

3. axiom $z$ is

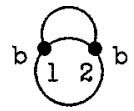

Productions $p_{1}$ and $p_{2}$ generate the two only connected planar maps of order 2 (label $b$ ensures applicability of these productions in the first step only). Production $P_{3}$ (from the preceding example) allows us to add loops on a vertex. Productions $p_{4}$ and $p_{5}$ allow us to cut off a vertex in two parts joined by a new edge (production $p_{s}$ (resp. $p_{4}$ ) is used when the two darts indicating the scission axis are joined (resp. not joined) by an edge).

3.4 Proposition: $L(G)$ is exactly the set of all connected planar maps.

Figure 4 shows an example of derivation sequence applied to the map issued from the previous example (dotted lines denote axis of scission).
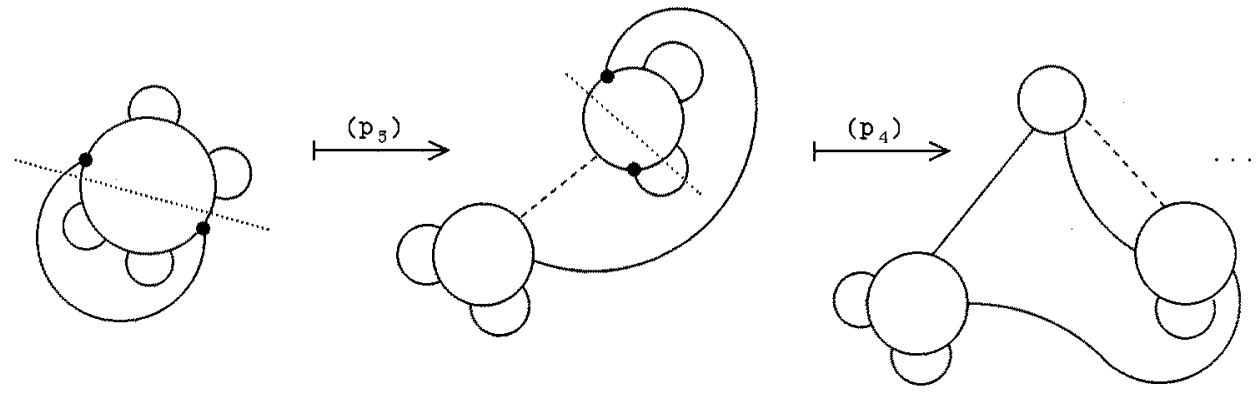

- figure 4 -

\section{HYPERMAP H-GRAMMARS}

In this section we deal with a particular subclass of hypermap grammars, called H-grammars, based upon hyperedge rewriting (note that a system based on vertex rewriting can be obtained as a dual form of H-grammars, acting on "dual hypermaps" $H^{\alpha}=(D, \alpha, \sigma, \lambda)$ ). We show that these grammars may be viewed as context-Free hypermap grammars, and exhibit a Pumping Theorem which generalizes a classical result from formal language theory.

We use in this section a ranked alphabet $\Sigma$ and denote by $\tau: \Sigma \rightarrow \mathbb{N}-\{0\}$ the rank function. This alphabet allows is to introduce the concept of H-hypermaps which is essential in our definition of H-grammars. 
4,1 Definition: A $H$-hypermap is a hypermap $H=(D, \sigma, \alpha, A)$ of $H_{z}$ verifying: for any hyperedge $C$ in $H$, for any dart $a$ in $C$, $\tau \lambda d=k$ where $k$ is the degree of $C$. Thus, the degree of any hyperedge in a H-hypermap is given by the labels of its darts.

4.2 Definition: A hypermap grammar $G=\langle\Sigma, \Delta, P, Z\rangle$ is a H-grammar iff:

(4.2.1) 2 is a H-hypermap.

$(4.2 .2)$ for every production $p=(L, R)$ we have:

- $L$ and $R$ are H-hypermaps,

- $\alpha_{I}$ is a circular permutation ( $L$ contains only one hyperedge),

$-\sigma_{R I D L}=\sigma_{L}$.

Condition $\sigma_{R \mid D b}=\sigma_{L}$ prohibits us from cutting off the vertices of a rewritten hypermap. The valuation of $\Sigma$ (and the concept of H-hypermaps) allows us to ensure the rewriting of full hyperedges.

As in the general case, we define notions of derivation step, derivation sequence and generated language of a H-grammar (note that all generated hypermaps are H-hypermaps). We call HHo-language any subset $I$ of HA such that there exists a H-grammar G verifying $L(G)=L$. The set of all $\mathrm{HH}_{\Delta}$-languages is denoted by LHH $_{\Delta}$.

The following lemma shows that the rewriting mechanism of H-grammars is strictly local and does not affect the context of rewritten hypermaps.

4.3 Lemma: Let $G=\langle\Sigma, \Delta, P, Z\rangle$ be a H-grammar, $\left\langle H, p, \mu, \xi, H^{\prime}\right\rangle$ a derivation step and $C_{0}$ the hyperedge of H rewritten by production $p$. Then, every hyperedge $C$ of $H$, distinct from $C_{0}$, remains unchanged in $H^{\prime}$ and we have $H_{1 \mathrm{C}}=H_{10}$.

This lemma induces the following corollary:

4.4 Corollary: Let $G=\langle\Sigma, \Delta, P, Z\rangle$ be a H-grammar; then we have:

$\left\{H_{10} / \exists H \in H_{z}, Z H \rightarrow H, C\right.$ hyperedge of $\left.H\right\}$

$\sim\left\{Z_{10} / C\right.$ hyperedge of $\left.z\right\} \cup\left\{R_{1} / \exists(L, R) \in P, C\right.$ hyperedge of $\left.R\right\}$

Note: Let $L_{1}$ and $L_{2}$ be two sets of hypermaps; by $L_{1} \sim L_{2}$ we mean:

$-\forall H_{1} \in L_{1}, \exists H_{z} \in L_{2}, H_{2} \sim H_{1}$,

$-\forall H_{2} \in L_{2}, \exists H_{1} \in L_{1}, H_{1} \sim H_{2}$.

Thus, every hyperedge in a hypermap derived from $Z$ is of bounded degree (the maximal degree is an integer $k_{0}$ depending on grammar $G$ only).

4.5 Remark: To avoid ambiguity when rewriting a given hypermap we will use the alphabet $\Sigma \times\left[k_{0}\right]$, where $k_{0}$ is the integer introduced in preceding corollary. Moreover, by using techniques from formal language theory, we can give any H-grammar $G$ in a normal form verifying: every hyperedge $C$ of axiom $z$ or right-hand-side $R$ of a production $p$ may be given as $c=\left(d_{1}, \ldots, d_{k}\right)$ such that:

i) $\pi_{1} \lambda a_{1}=\ldots=\pi_{1} \lambda d_{k}=a \in \Sigma, \tau a=k$,

ii) if $c^{\prime}=\left(d_{1}^{\prime}, \ldots, d_{k}^{\prime}\right)$ is another such hyperedge of same degree than $c$ 
$H_{\mathrm{n}}=I \operatorname{ter}\left(H, M, E_{\mathrm{M}}, n\right)$ as follows:

i) $H_{0}=H$,

ii) $H_{1}=\left(D_{1}, \sigma_{1}, \alpha_{2}, \lambda_{1}, A_{1}\right)$,

with $\left(D_{1}, \sigma_{1}, \alpha_{1}, \lambda_{1}\right)=F_{\alpha}\left(H_{0}, M, E_{M}\right)=\xi_{1} M \otimes H_{01}^{-1} \otimes H_{0}$

and $A_{\lambda}=\xi_{1} A_{0}$,

iii) for every $t \geqslant 1, H_{\mathrm{t}}=\operatorname{Iter}\left(H_{\mathrm{t}-1}, M, E_{\mathrm{X}}, 1\right)$.

Hypermap $H$ (resp. $M$ ) is called base (resp. pattern) of the iteration.

4.10 Example: Figure 5 shows two hypermaps $H$ and $M$ and the two first corresponding iterations $H_{1}=I$ ter $\left(H, M, E_{M}, 1\right)$ and $H_{2}=I$ ter $\left(H, M, E_{M}, 2\right)$.

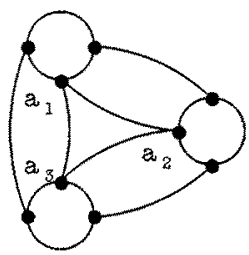

base $\mathrm{H}$

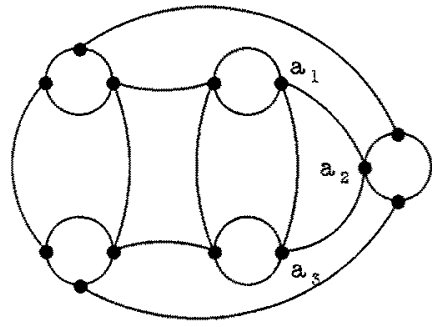

$\operatorname{Iter}\left(\mathrm{H}, \mathrm{M}, \mathrm{E}_{\mathrm{M}}, \mathrm{I}\right)$

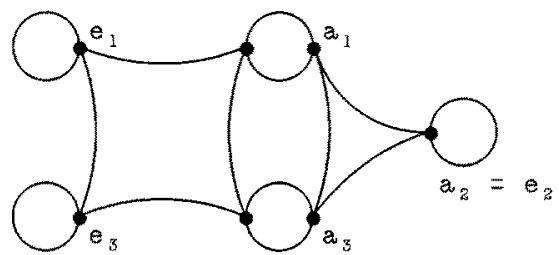

pattern M

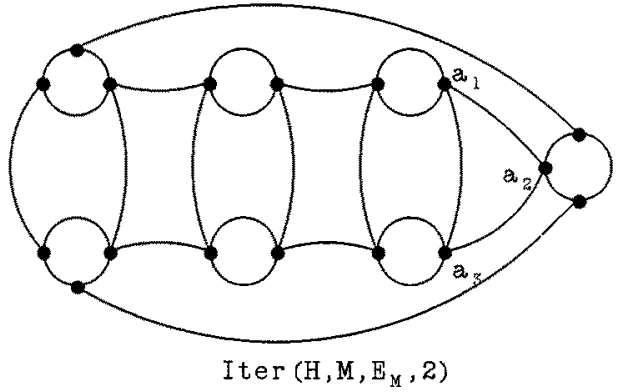

- figure 5 -

4.11 Theorem (Pumping Theorem): Let $G=\langle\Sigma, \Delta, P, Z\rangle$ be a H-grammar such that $L(G)$ is infinite. Then, there exists two positive integers $K 1$ and K2 verifyine: for every hypermap $H$ in $L(G)$ such that \#D $>K 1$, there exists a positive integer $k$, a base. $X$ and a pattern $M$, an H-hypermap $Y$ and a sequence $E_{Y}$ of $k$ distinct darts of $Y$ such that:

(4.11.1) \#D $D_{\mathrm{M}}+\# D_{\mathrm{Y}} \leqslant K 2$,

(4.11.2) \#D $\geqslant k$,

(4.11.3) $H=F_{\text {a }}\left(I \operatorname{ter}\left(X, M, E_{\mathrm{M}}, 1\right), Y, E_{Y}\right)$,

(4.11.4) $\forall n \geqslant 0, F_{\alpha}\left(I \operatorname{ter}\left(X, M, E_{\mathrm{M}}, n\right), Y, E_{X}\right) \in I(G)$.

This situation is depicted below:

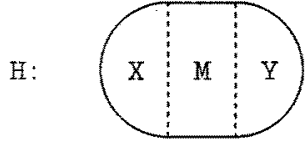

and
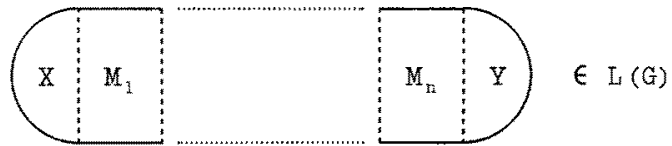
but of different "shape" (i.e, $\sigma_{1} \neq \sigma_{1}$, ) then $\pi_{1} \lambda d_{1} \neq \pi_{1} \lambda d_{1}^{*}$, ii $i$ ) $\pi_{2} \lambda a_{i}=i$ for any $i$ in $[k]$.

where $\pi_{1}$ and $\pi_{2}$ denote respectively projections on first and second component.

We assume now that alphabet $\Sigma$ is such that every H-grammar $G$ can be Eiven in normal form.

For every hyperedge in a hypermap derived from $z$, the labels of its darts determine its degree but also its shape (\#I represents the number of different hyperedge shapes (including their degree) that may appear in a HH-language).

The following lemma indicates that H-grammars verify a property of Church-Rosser type.

4.6 Lemma: Let $H=(D, \sigma, \alpha, \lambda)$ be an H-hypermap, $p_{1}$ and $p_{2}$ two productions which may be applied to $H$ over two distinet hyperedges $C_{1}$ and $C_{2}$; we know (lemma 4.3) that if $H$ directly derives $H_{1}$ (resp. $H_{2}$ ) by application of $p_{1}$ (resp. $p_{3}$ ), then $p_{2}$ (resp. $p_{1}$ ) may be applied in $H_{1}$ (resp. $H_{2}$ ) to the same hyperedge $C_{z}$ (resp. $C_{1}$ ). Moreover, we have:

if $H \mapsto_{\mathrm{p} 1} H_{3} \mapsto_{\mathrm{p} 2} H_{12}$ and $H \mapsto_{\mathrm{p} 2} H_{2} \mapsto_{p_{1}} H_{23}$, then $H_{12} \sim H_{Z_{1}}$.

In our pumping theorem, we will use the following construction, based on the concept of $\alpha_{k}$-pointed hypermaps, to desoribe the structure of HH-languages.

4.7 Definition: A $\alpha_{k}$-pointed hypemap is a 5 -tuple $H=(D, \sigma, \alpha, \lambda, A)$ where $H=(D, \sigma, \alpha, \lambda)$ is a H-hypermap and $A$ a sequence of $k$ distinct darts $a_{1}, \ldots, a_{k}$ such that $\left(a_{1}, \ldots, a_{k}\right)$ is a hyperedge and $\pi_{2} \lambda a_{i}=i, \forall i \in[k]$.

4.8 Definition: Let $H=(D, \sigma, \alpha, \lambda, A)$ be a $\alpha_{k}$-pointed hypermap, $H^{\prime}=\left(D^{\prime}, \sigma^{\prime}, \alpha^{\prime}, \lambda^{\prime}\right)$ a hypermap such that $D^{\prime} \cap D=\varnothing$, and $E^{\prime}=e_{1}^{\prime}, \ldots, e_{\mathrm{k}}^{\prime}$ a sequence of $k$ distinct darts of $H^{*}$. We call $\alpha$-fusion along $E^{\prime}$ of hypermaps $H$ and $H^{\prime}$, denoted $F_{a}\left(H, H^{\prime}, E^{\prime}\right)$ the hypermap defined over (DUD') $\backslash E^{\prime}$ by :

$$
F_{*}\left(H, H^{\prime}, E^{\prime}\right)=\xi H^{\prime} \otimes H_{1 A}^{-1} \otimes H,
$$

where $\xi$ is the mapping from $D^{\prime}$ to $\left(D^{\prime} \cup A\right) \backslash E^{\prime}$ defined by: $\forall a \in D^{\prime}$, if $a=e_{i}^{\prime}$ then $\xi d=a_{i}$ else $\xi d=d$.

This operation is only a slightly different formulation of the hyperedge rewriting mechanism we have defined (if $G=\langle\Sigma, \Delta, P, Z\rangle$ is a H-grammar and $\left\langle H, p, H, \xi, H^{x}\right\rangle$ a derivation step, then we have $H^{\prime}=$ $F_{\alpha}\left(H, R,\left\{1, \ldots, \# D_{\mathrm{t}}\right\}\right)$.

The notion we introduce now allows us to generalize to hypermas the concept of concatenation from formal language theory.

4.9 Definition: Let $H=\left(D_{H}, \sigma_{H}, \alpha_{H}, \lambda_{H}, A_{H}\right)$ and $M=\left(D_{M}, \sigma_{M}, \alpha_{M}, \lambda_{M}, A_{M}\right)$ be two

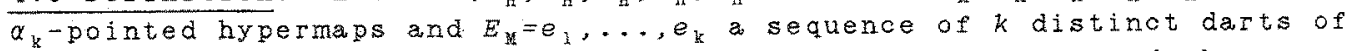
$D_{M}$. For any integer $n \geqslant 0$, we define the $\alpha_{k}$-pointed hypermap 
Theorem 4.11 induces the following corollary:

4.12 Corollary: Let $G=\langle\Sigma, \Delta, P, Z\rangle$ be a H-grammar such that $L(G)$ is infinite; let $N B(G)=\left\{n \in \mathbb{N} / \exists H \in I(G), \# D_{\mathrm{H}}=n\right.$, Then, there exists integers $n_{0}$ and $k$ such that: $\forall i>n_{0},[i, i+k] \cap N B(G) \neq \varnothing$.

This corollary enable us to state that there exists hypermap families that are not HH-languages. It is namely the case for the following:

4.13 Example: The set of all hypermaps of the following form cannot be Benerated by an H-grammar:
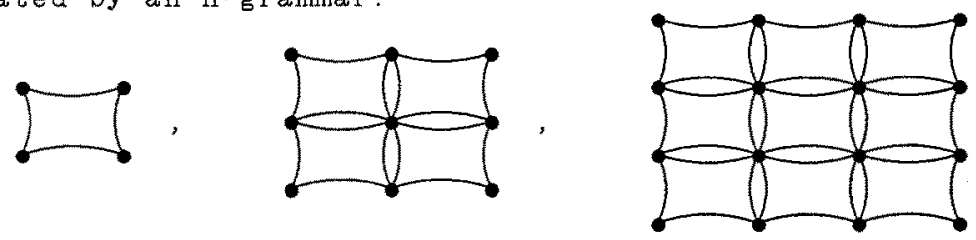

The following theorem shows that H-grammars allow us to retrieve hypergraph languages generated by hyperedge replacement, introduced by Habel and Kreowski [7]. We will denote by HK-grammar such a system.

4.14 Theorem: For any HK-grammar HKG, there exists a H-grammar HG such $\overline{\text { that: } L(H K G)}=U n d(L(H G))=\{U n d(H) / H \in L(H G)\}$.

Acknowledgements: I would like to thank Prof. B. Courcelle for many helpful suggestions with respect to the final version of this paper.

References:

[1] M. BAUDERON, B. COURCELLE, "Graph Expressions and Graph Rewritings", Rapport interne $n^{\circ} I-8623$, Université de Bordeaux $I$, Sept. 1986 .

[2] V. CLAUS, H. EHRIG, G. ROZENBERG, "Graph Grammars and their Application to Computer science and Biolagy", LNCS 73, Springer-Verlag, 1980 .

[3] R. CORI, "Un code pour les graphes ptanaires et ses applications", Astérique 27,1975 .

[4] J. EDMONDS, "A Combinatorial Representation for Polyhedral surfaces", Not. Amer. Math. Soc. 7, 1960.

[5] H. EHRIG, "Introduction to the Algebraic Theory of Graph Grammars", LNCS 56, 1977, pp. 245-255.

[6] H. EHRIG, M. NAGL, G. ROZENBERG, "Graph Grammars and their Application to Computer science", LNCS 153, Springer-Verlag, 1983.

[7] A. HABEL, H.J. KREOWSKI, "Some structural Aspects of Hypergraph Languages Generated by Hyperedge Replacement", Preprint, 0ct. 1985.

[8] D. JANSSENS, "Node-Label-Controlled Graph Grammars", Ph.D Thesis, Univ. of Antwerpen, 1983.

[9] E. SOPENA, "Récriture d'hypercartes combinatoires et conception d'un langage pour la programmation d'algorithmes de graphes". Thèse de Dootorat, Université de Bordeaux I, Nov. 1986. 